\title{
A Study to Assess the Prevalence and Contributing Factors of Ventilator-associated Pneumonia among Patients Admitted to Critical Care Units, MGMCRI, from January to December 2019
}

\author{
M Malarmathi ${ }^{1} \oplus$, S Nandhini ${ }^{2} \oplus$, Lavanya $^{3}$, K Renuka $^{4}$
}

\begin{abstract}
Background: Ventilator-associated pneumonia (VAP) is the most frequent life-threatening nosocomial infection in intensive care units (ICUs). Reported incidences vary widely from 5 to $40 \%$ depending on the setting and diagnostic criteria.

Aims and objectives: (1) To assess the prevalence of VAP among patients admitted to critical care units. (2) To find out the contributing factors of VAP among patients admitted to critical care units.

Materials and methods: A retrospective approach was adopted for the study. The population of the study involves the patients diagnosed with VAP. The cluster sampling technique was used. Data were collected from the records based on the demographic variables and the contributing factors that were listed out in the study.

Results: With regard to the contributing factors of the VAP, 40 samples were available. Out of these 40 samples, 12 (30\%) were with respiratory illness, 7 (17.5\%) were with cardiovascular disorders, 7 (17.5\%) were with kidney diseases, 5 (12.5\%) were with prolonged length of stay, 2 (5\%) were with the resistance to antibiotics and invasive procedures, $3(7.5 \%)$ were with trauma, and $1(2.5 \%)$ was with septicemia and multiple organ dysfunction syndrome.

Keywords: Contributing factors, Prevalence, Ventilator-associated pneumonia.

Pondicherry Journal of Nursing (2021): 10.5005/jp-journals-10084-13103
\end{abstract}

\section{INTRODUCTION}

Respiration is one of the essential processes required for survival, as well as for providing the energy required for all vital life processes. It is the process of exchange of gases through inhalation and exhalation of respiratory gases that are essential functions of the respiratory system in the human body. ${ }^{1}$ Respiratory diseases are widespread rapidly, and they are considered the world's fifth deadly disease. In India, respiratory disorders, such as chronic obstructive pulmonary disease, asthma, pneumonia, tuberculosis, and interstitial lung disease, are ranked as the third leading cause of death. ${ }^{2}$ Pneumonia is the second most common nosocomial infection in critically ill patients, affecting $27 \%$ of patients admitted to critical care units. ${ }^{3}$

When pneumonia develops for more than 48 hours after a patient's admission to the hospital and is not incubating at the time of admission, it is classified as a nosocomial infection. ${ }^{4}$ About $86 \%$ of pneumonias are ventilator-associated pneumonia (VAP), which are caused by mechanical ventilation. ${ }^{5}$

The Centers for Disease Control and Prevention standard for surveillance is the rate of cases per 1000 ventilator days. The prevalence of VAP ranges from $28 \%$ to $32 \%$ of patients. ${ }^{6}$ In India, it affects $9 \%$ to $27 \%$ of intubated patients and doubles the risk of death as compared to patients who do not have VAP.

Adult cases of VAP are estimated to number 4000 a year, resulting in 230 deaths, 17,000 days in the intensive care units (ICUs), and 46 million in healthcare costs. In order to avoid the VAP, nurses are the first line of protection. It shows that together with
${ }^{1,2}$ Department of Medical Surgical Nursing, Kasturba Gandhi Nursing College, Puducherry, India

${ }^{3}$ Department of Obstetrics and Gynecology Nursing, Kasturba Gandhi Nursing College, Puducherry, India

${ }^{4}$ Department of Medical Surgical Nursing, Kasturba Gandhi Nursing College, Sri Balaji Vidyapeeth, Puducherry, India

Corresponding Author: S Lavanya, Department of Obstetrics and Gynecology Nursing, Kasturba Gandhi Nursing College, Puducherry, India, Phone: +91 9994747440, e-mail: lavanyas@kgnc.ac.in

How to cite this article: Malarmathi M, Nandhini S, Lavanya S, Renuka K. A Study to Assess the Prevalence and Contributing Factors of Ventilator-associated Pneumonia among Patients Admitted to Critical Care Units, MGMCRI, from January to December 2019. Pon J Nurs 2021;14(2):26-28.

Source of support: Nil

Conflict of interest: None

other health-care workers, nurses have a vital role in preventing VAP because many of the procedures were done by them in dayto-day nursing care. ${ }^{7,8}$

\section{Овjectives}

- To assess the prevalence of VAP among patients admitted to critical care units.

- To find out the contributing factors of VAP among patients admitted to critical care units.

(0) The Author(s). 2021 Open Access This article is distributed under the terms of the Creative Commons Attribution 4.0 International License (https:// creativecommons.org/licenses/by-nc/4.0/), which permits unrestricted use, distribution, and non-commercial reproduction in any medium, provided you give appropriate credit to the original author(s) and the source, provide a link to the Creative Commons license, and indicate if changes were made. The Creative Commons Public Domain Dedication waiver (http://creativecommons.org/publicdomain/zero/1.0/) applies to the data made available in this article, unless otherwise stated. 


\section{Materials and Methods}

A retrospective approach was adopted for the study. The study was conducted in critical care units in MGMCRI, Puducherry. The cluster sampling technique was used. The population of the study includes patients diagnosed with VAP and who got admitted to MGMCRI, from January to December 2019. Data were collected from the records based on the demographic variables and the contributing factors that were listed out in the study.

\section{Criteria for Sample Selection Inclusion Criteria}

- Patients with age-group between 20 and 60 years.

- Patients who received mechanical ventilation.

- Both male and female patients.

\section{Exclusion Criteria}

- Patients after 24 hours of intubation.

- Patients already diagnosed with fever, pneumonia, and acute respiratory distress syndrome.

- Patients with cervical and spinal cord injury.

\section{Results}

Frequency and Distribution of Demographic Variables of Patients with Ventilator-associated Pneumonia

With regard to age, the maximum 20 (50\%) were from 41 to 50 years. In gender, 24 (60\%) were male. In religion, 30 (75\%) were Hindus. In the area of residence, 34 (85\%) were residing in rural areas. In the type of diet, $16(40 \%)$ were nonvegetarian. With regard to income, $29(72.5 \%)$ were from Rs. 5000 to 10,000 . With regard to work, 25 $(62.5 \%)$ were doing the moderate type of work. In primary medical diagnosis, 12 (30\%) were having a pulmonary disorder. In ICU length of stay, the maximum of $12(30 \%)$ were from 7 to 10 days. The duration of mechanical ventilation of 21 (52.5\%) patients was between 7 and 10 days. With regard to the frequency of suctioning, $20(50 \%)$ were suctioned at third hourly (Fig. 1).

\section{Frequency and Percentage Distribution of Total Number of Patients with Ventilator-associated Pneumonia from January to December 2019}

\begin{tabular}{llll}
\hline & Total number of patients \\
admitted to critical care & Total number of & \\
units, MGMCRI, from January & patients with VAP & \\
from January to & Prevalence \\
S. No. & to December 2019 & December 2019 & rate \% \\
\hline a. & 1220 & 40 & 2.51 \\
\hline
\end{tabular}

\section{Frequency and Distribution of Contributing Factors of Patients with Ventilator-associated Pneumonia}

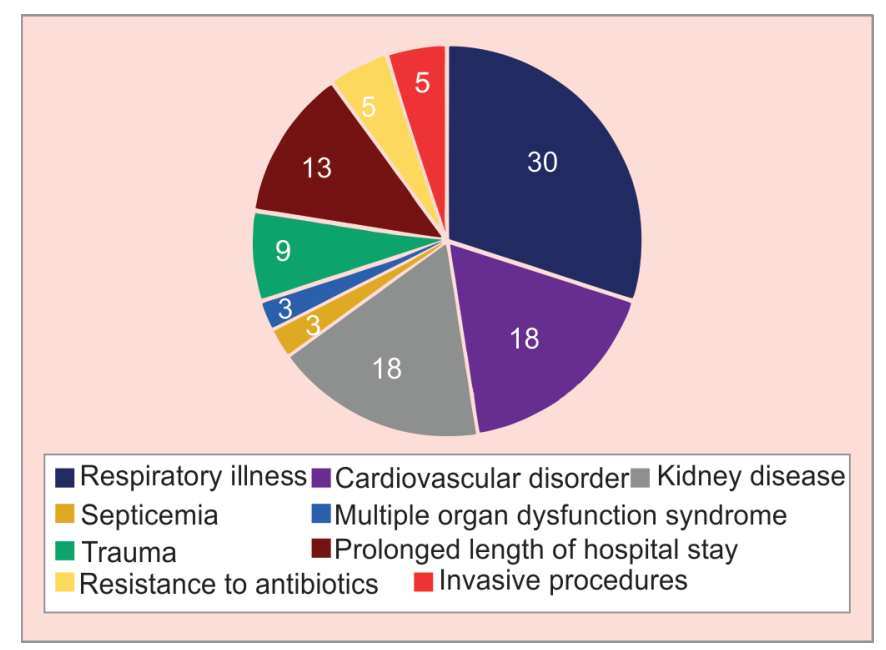

Fig. 1: The study result shows the contributing factors of VAP. A total of 40 samples were available. Out of these 40 samples, 12 (30\%) were with respiratory illness, $7(17.5 \%)$ were with cardiovascular disorders, 7 (17.5\%) were with kidney diseases, 5 (12.5\%) were with prolonged length of stay, $2(5 \%)$ were with the resistance to antibiotics and invasive procedures, $3(7.5 \%)$ were with trauma, and 1 (2.5\%) was with septicemia and multiple organ dysfunction syndrome

\section{Discussion}

The first objective of the present study was to assess the prevalence of VAP among patients admitted to critical care units in MGMCRI, from January to December 2019. There were a total of 1220 cases; out of these, 40 (2.51\%) patients were diagnosed with VAP.

The second objective of the present study was to find out the contributing factors of VAP.

VAP represents a high percentage of ICU-acquired infections and is associated with significant antibiotic utilization in the ICU and substantial morbidity, mortality, and costs. VAP cases are potentially preventable. The ventilator bundle (head of bed elevation, oral hygiene, patient mobility, and coughing and deep breathing) has been an effective intervention for VAP prevention. ${ }^{7}$ As a result, studies are required to assess the bundles that include interventions by the most recent recommendations, to see whether they have an impact on patient's health outcomes and VAP incidence reduction when implemented together. 


\section{ConcLusion}

The prevalence of VAP exists as an enormous burden in the society. This study concludes the prevalence of VAP among patients admitted to critical care units. Patients may be treated medically or surgically depending on the cause, resulting in a higher cure and success rate for the patients.

\section{OrCID}

M Malarmathi (1) https://orcid.org/0000-0003-2224-1166

S Nandhini (i) https://orcid.org/0000-0001-5691-9452

\section{References}

1. Arlene LP. Core principle and practice of medical surgical nursing. 2nd ed. Haryana: Elsevier Publication, 2010.

2. Barbara K, Timby N, Smith E. Introduction to medical surgical nursing. 9th ed. Philadelphia: Lippincott Williams and Wilkin, 2007.

3. Richards MJ, Edwards JR, Culver DH, et al. Nosocomial infections in medical intensive care units in the United States. Crit Care Med 1999;27(5):887-892. DOI: 10.1097/00003246-199905000-00020.
4. SheblE, Gulick PG. Nosocomial pneumonia. Treasure Island: Stat Pearls, 2020.

5. American Thoracic Society, Infectious Diseases Society of America. Guidelines for the management of adults with hospital-acquired, ventilator-associated, and healthcare-associated pneumonia. Am J Respir Crit Care Med 2005;171(4):388. DOI: 10.1164/rccm.200405644ST.

6. Tao L, Hu B, Rosenthal VD, et al. Impact of a multidimensional approach on ventilator-associated pneumonia rates in a hospital of Shanghai: findings of the International Nosocomial Infection Control Consortium. J Crit Care 2012;27(5):440-446. DOI: 10.1016/ j.jcrc.2011.12.018.

7. De Miguel-Díez J, Jiménez-García R, Hernández-Barrera V, et al. Trends in hospitalizations for community-acquired pneumonia in Spain: 2004 to 2013. Eur J Internal Med 2017;40:64-71. DOI: 10.1016/ j.ejim.2016.12.010.

8. Wip C, Napolitano L. Bundles to prevent ventilator-associated pneumonia: how valuable are they? Curr Opin Infect Dis 2009;22(2):159-166. DOI: 10.1097/QCO.0b013e3283295e7b. 